\title{
Features and behavior of valvular abnormalities in adolescent and adult patients in mucopolysaccharidosis: an echocardiographic study
}

\author{
Domenico Galzerano ${ }^{1,2 *}$, Seemi Saba ${ }^{*}$, Abdullah Al Sergani ${ }^{3}$, Olga Vriz ${ }^{1,2}$, Khalil Alghalayini ${ }^{3}$, \\ Khushnooda Ramzan ${ }^{4}$, Isra Elmahi ${ }^{2}$, Antonio Cittadini ${ }^{5}$, Giovanni Di Salvo ${ }^{6}$, Valeria Pergola ${ }^{7}$ \\ *Equally first author
}

${ }^{1}$ King Faisal Specialist Hospital \& Research Centre, The Heart Centre, Riyadh, Saudi Arabia; ${ }^{2}$ College of Medicine, Alfaisal University, Riyadh, Saudi Arabia; ${ }^{3}$ King Saud University, College of Medicine, Riyadh, Saudi Arabia;

${ }^{4}$ Department of Genetics and Research Centre, King

Faisal Specialist Hospital \& Research Centre, Riyadh, Saudi Arabia; ${ }^{5}$ Department of Translational Medical

Correspondence: Prof. Domenico Galzerano, M.D., FESC, FEACVI, Professor of Medicine, Alfaisal University, College of Medicine, Consultant Cardiology, The Heart Centre, King Faisal Specialist Hospital \& Research Center, Riyadh, Saudi Arabia.

Tel. +966.539037762. Fax: +966.11.4427791.

E-mail: domenicogalzerano@libero.it

Key words: Mucopolysaccharidoses; echocardiography; valvular disease.

Acknowledgments: We would like to thank Fatima Arshi, RCS, for the master collaboration in performing the studies, and Kristel Sumagaysay for her technical assistance in writing the manuscript.

Authors' contributions: DG, began the project, collected data, wrote, reviewed the manuscript and the figures, looked for and examined the references and approved the final manuscript; SS, began the project, collected data, wrote, reviewed the manuscript and the figures, looked for and carefully examined the references and approved the final manuscript; AAS, collected data, wrote, reviewed, and approved the final manuscript; $\mathrm{OV}$, interpreted and commented echocardiographic imaging, wrote, reviewed and approved the final manuscript; KA, collected clinical data, wrote, reviewed the manuscript and approved the final manuscript; KR, interpreted and commented clinical results, wrote, reviewed and approved the final manuscript; IE, collected clinical data, wrote, reviewed the manuscript and the figures, looked for and carefully examined the references and approved the final manuscript; AC, interpreted and commented echocardiographic imaging, wrote, reviewed and approved the final manuscript; GDS, interpreted and commented clinical results, wrote, reviewed and approved the final manuscript; VP, interpreted and commented clinical results and echocardiographic imaging, wrote, reviewed and approved the final manuscript.

Conflict of interest: The authors declare that they have no competing interests, and all authors confirm accuracy.

Ethics Approval: The study was approved by the Ethical Committee of our Institution (approval number: RAC 2201151).

Availability of data and materials: The datasets used and/or analyzed during the current study are available from the corresponding author on reasonable request.

Received for publication: 13 January 2021.

Accepted for publication: 27 May 2021.

(C) Copyright: the Author(s), 2021

Licensee PAGEPress, Italy

Monaldi Archives for Chest Disease 2021; 91:1767

doi: 10.4081/monaldi.2021.1767

This article is distributed under the terms of the Creative Commons Attribution Noncommercial License (by-nc 4.0) which permits any noncommercial use, distribution, and reproduction in any medium, provided the original author(s) and source are credited.

Sciences, Federico II University, Naples, Italy;

${ }^{6}$ Paediatric Cardiology and Congenital Heart Disease

Department, University of Padua, Italy; ${ }^{7}$ Department of Cardio-Thoraco-Vascular Sciences and Public Health, University of Padua, Italy

\section{Abstract}

Mucopolysaccharidoses, a rare inherited disorder of lysosomal storage, account for less than $0.1 \%$ of all genetic diseases. The penetrance is highly variable and clinically it varies from severe fetalneonatal forms to attenuated diseases diagnosed in adult individuals. The majority of the patients have been reported to show cardiac abnormalities since pediatric age, however, there is a minority of patients with attenuated disease diagnosed in the adolescent and adult age. The haematopoietic stem cell transplantation and enzyme replacement therapy are the current therapies for these disorders. Thanks to these treatments, Mucopolysaccharidoses patients live longer than in the past. Even though enzyme replacement therapy has been demonstrated to reduce left ventricular mass in patients with cardiomyopathy, the efficacy on valve abnormalities has not been clearly demonstrate yet. Furthermore, thanks to the current therapy, to better understanding of the disease and to the advent on new technologies, an increasing number of adolescent and adult patients diagnosed with MPS are followed up in the adult echocardiographic laboratory. Indeed, a systematic descriptive study describing the echocardiographic features of valvular involvement and their evolution in adolescent and adult patients lacks of medical literature and this was the aim of our investigation. Our results showed that all the valves are affected, mainly the mitral valve with a higher prevalence compared to the pediatric age. The echocardiographic features of MPS differs from other valvular disease of adolescent and adult age, and knowing them can avoid misdiagnosis. Our observations also suggest that the progression of cardiac involvement slows after the initiation of the therapy in our group of age. Further studies on larger population are required to confirm our results.

\section{Introduction}

Mucopolysaccharidoses (MPS) are inherited disorders of lysosomal storage that are caused by functional enzymes deficiency which are responsible for the degradation of glycosaminogly- 
cans (GAG) leading to the progressive systemic deposition of GAG and dysfunction of multi-organ system that differs with the particular deposited GAG and the present of the specific enzyme mutations [1,2].

MPS account for less than $0.1 \%$ of all genetic diseases [1,2]. There are 7 different types, I, II, III, IV, VI, VI and IX. according to the different involvement [1-4]. The penetrance is highly variable and clinically it varies from severe fetal-neonatal forms to attenuated diseases diagnosed in adult individuals. The majority of the patients have been reported to show cardiac abnormalities since pediatric age, however, there is a minority of patients with attenuated disease diagnosed in the adolescent and adult age $[1,2]$. Cardiac structural abnormalities are present in all types of MPS, but more commonly in MPS types I, II and VI. MPS lead to the accumulation of glycosaminoglycan in various cardiovascular structures. It can cause valvular abnormalities, myocardial dysfunction, coronary artery disease, arrhythmias, and aortopathy. The hematopoietic stem cell transplantation (HSCT) and enzyme replacement therapy (ERT) are the current therapies for these disorders [1-4]. Thanks to these treatments, MPS patients live longer than in the past. Even though ERT has been demonstrated to reduce left ventricular mass in patients with cardiomyopathy $[3,4]$, the efficacy on valve abnormalities has not been clearly demonstrate yet. The most common causes of death include respiratory failure and cardiac failure [5]. Echocardiography is considered gold standard noninvasive imaging modality for detecting cardiac abnormalities in MPS $[1,2,3,6]$. However, in the mild forms, echocardiographic features of MPS can be misdiagnosed with other cardiac disease as rheumatic heart disease and myxomatous valves. In pediatric age previous reports have showed in the early stage valvular thickening with regurgitation, while in the late stage there are worsening of fibrosis and calcification leading to valve stenosis. Intramyocardial infiltration of pseudohypertrophy (GAGs) is rarely reported compared to other storage diseases [1-5]. In this scenario, novel echocardiographic tools such as strain, strain rate and speckle tracking technologies have shown to be useful to detect subclinical myocardial functional abnormalities $[3,4,6]$

Furthermore, thanks to the current therapy, to better understanding of the disease and to the advent on new technologies, an increasing number of adolescent and adult patients diagnosed with MPS are followed up in the adult echocardiographic laboratory.

Indeed, a systematic descriptive study describing the echocardiographic (E) features of valvular involvement and their evolution in adolescent and adult patients lacks of medical literature and this was the aim of our investigation.

\section{Materials and Methods}

Inclusion criteria: Patients diagnosed with MPS through enzymatic or genetic examinations undergoing transthoracic echocardiography at our echocardiographic laboratory were included.

Exclusion criteria: poor acoustic window.

Study design: observational descriptive retrospective study.

Age: all patients from 14 years and up have been included in our study.

\section{Echocardiography}

Individual measurements were made from optimal still frames of cine-loops. Measurements will be made in millimeters to an accuracy of two decimal places as per machine settings. All the standard measurements have been performed according to American Society of Echocardiography (ASE) and European
Association of Cardiovascular Imaging (EACVI) guidelines on chamber quantification 2015 [8].

Mitral valve leaflets thickness, mobility and calcification and involvement of subvalvular apparatus, aortic valve leaflets thickness and tricuspid valve leaflets thickness were measured.

\section{Thickening of valve leaflets}

A large autopsy bases study on a 200-patient group determined the various ranges of thickness in various age groups [9]; valve thickness increased with age, anterior mitral valve leaflet AMVL thickness was $1.3 \pm 0.5 \mathrm{~mm}$ in 40 subjects under 20 years of age, $1.6 \pm 0.85 \mathrm{~mm}$ in subjects $20-59$ years of age and $3.2 \pm 1.52 \mathrm{~mm}$ in subjects 60 years and older. They reported a mean free edge measurement of aortic valve was $0.67 \mathrm{~mm} \pm 0.21 \mathrm{~mm}$ (range $0.35-1.55 \mathrm{~mm}$ ) in 40 hearts from individuals $<20$ years. Another study among children to determine the normal ranges of valve thickness reveal in normal children, median AMVL tip thickness was $2.0 \mathrm{~mm}$ (IQR 1.7-2.4) and median AMVL midpoint thickness $2.0 \mathrm{~mm}$ (IQR 1.7-2.4). The median aortic valve thickness was $1.5 \mathrm{~mm}$ (IQR 1.3-1.6) in the parasternal long axis view and $1.4 \mathrm{~mm}$ (IQR 1.2-1.6) in the parasternal view [10].

To the best of our knowledge no data dealing tricuspid valve and pulmonic valve thickness in healthy patients have been previously reported. According to these previous investigations, we considered as thickened the leaflets when the MV leaflets were $>2$ $\mathrm{mm}$ of thickness and when the aortic and tricuspid valve leaflets were $>1.5 \mathrm{~mm}$.

\section{Mitral valve}

MV leaflets thickness: anterior mitral valve leaflet (AMVL) thickness was measured at the midpoint in the clear zone and at the tip of the leaflet in the parasternal long axis (PSLA) view in diastole, when the AMVL will be approximately parallel to the ventricular septum (Figure 1A). This will be usually in the still frame of maximal diastolic excursion of the AMVL. Measurements of the [posterior mitral valve leaflet (PMVL)] thickness was measured at the midpoint in diastole in a still frame where there will be clear separation of the PMVL from other posterior annulus and myocardial wall (Figure 1A).

MV leaflets mobility: The mobility of the leaflets of mitral valve was defined as normal or reduced when it was not possible to achieve a position parallel during diastole for the anterior mitral leaflet to the interventricular septum and for the posterior mitral leaflet to posterolateral wall in parasternal long axis view. The mobility for the anterior mitral leaflet was further assessed for the marginal or mid segments or involving the whole leaflets. The mobility of posterior mitral leaflet was categorized as normal and reduced in the parasternal long axis view.

Calcification was diagnosed as present when an increased area of echogenicity of white color was found and defined as focal when a well circumscribed area was detected or extended when involving more than the marginal part or the mid part or the basal part of the anterior mitral leaflet. The calcification of posterior mitral leaflet will be categorized as absent or present in the parasternal long axis view.

Subvalvular apparatus involvement will be defined as presence of thickening or fusion in the area adjacent to MV leaflets or extending to the papillary muscles.

\section{Aortic valve}

The thickness of right coronary cusp (RCC) and non-coronary cusp (NCC) was measured from PSLA view in systole when the cusps were at maximum excursion (Figure 1B). 


\section{Tricuspid valve}

The thickness, mobility, calcification of the leaflet of tricuspid valve attached to the myocardial wall in 4 chamber view in the early diastolic phase when the ultrasound beam is almost perpendicular to the leaflet were assessed; in the majority of the case the leaflet imaged in this view is the anterior. Nevertheless, the leaflets identification from this view is a matter of great controversy due to variability of TV anatomy (Figure 1C).

\section{Pulmonic valve}

The pulmonic valve was visualized in short axis view at the level of the great vessels. Function of the valves was assessed according to the ASE, EACVI, and European Society of Cardiology (ESC) guidelines $[7,8,11]$. We considered in our study

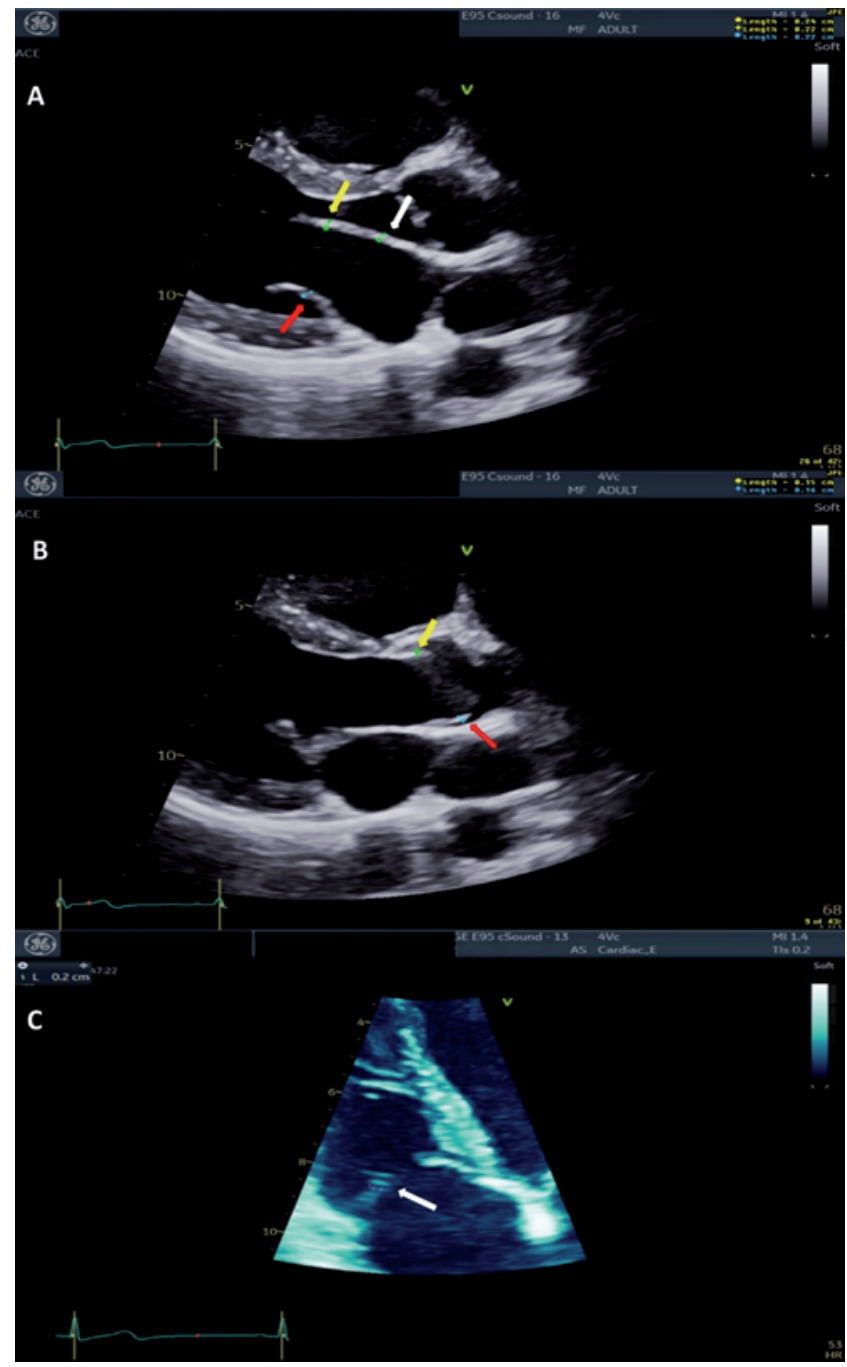

Figure 1. Two-dimensional echocardiography, valve leaflets measurement (see details in the text). A) Mitral valve leaflets measurements, parasternal long axis view in diastole, anterior mitral valve leaflet midpoint (white arrow), anterior mitral valve leaflet tip (yellow arrow), posterior mitral valve leaflet midpoint (red arrow). B) Aortic valve measurements on parasternal long axis view in systole, right coronary cusp (yellow arrow), noncoronary cusp (red arrow). C) Tricuspid valve measurements, four chamber view in early diastolic phase, (white arrow): anterior tricuspid leaflet. a valve dysfunction at least a moderate degree of regurgitation and a mild stenosis were detected.

\section{Statistical analysis}

Statistical analysis and estimates were performed using the Statistical Package for the Social Sciences (SPSS) software v. 24.0 for Windows (SPSS Inc, IL, USA) and Microsoft Excel 2017. Categorical variables were evaluated by Chi-square test and continuous values were compared using paired Student's $t$-test. Statistical significance was set at $\mathrm{p}<0.05$.

\section{Results}

In Table 1 are illustrated the general characteristics of the population. Our study group is composed of 17 adolescent and adult patients affected by MPS with age range from 15 to 48 years. Mostly of pts were male, affected by type 6 MPS, receiving therapy with ERT. Follow up duration ranged from 1 to $18 \mathrm{yrs}$; during this period the patients underwent 46 echo studies. Table 2 shows the echocardiographic features regarding thickening, valve motion, calcification of the mitral, aorta and tricuspid valves (Figure 2). The most frequently affected valve was the MV, showing both leaflets thickening in $88 \%$ of patients and reduced valve mobility in $63 \%$ of the patients. AMVL reduced mobility involved mainly the whole leaflet more than being isolated at the tip or the midpoint since the earlier stage. Same applies for the PMVL. Aortic and tricuspid valves were also frequently involved, showing an analogue pattern with thickening of $76 \%$ on the aortic valve and $82 \%$ on the tricuspid valve. The pulmonic valve leaflets are very thin and were difficult to correctly visualize and measure. However, in 4 cases a clear thickening of the valve was visually noted. The functional assessment of the valves is illustrated in Table 3. The stenosis was the predominant dysfunction of the MV being present $52 \%$ of the patients as isolated in 29 and as associated with at least moderate regurgitation in $23 \%$ of the patients. Different results were found on the other valves: at least moderate regurgitation was the most frequent aortic and tricuspid valve dysfunction being present in $34 \%$ of the patients (29 isolated and $5 \%$ associated with stenosis) and in the $23 \%$ respectively. The behavior of the valve thickness over the years of follow up is reported in Table 4. No significant statistical difference in valve thickness from the baseline measurement at younger age when compared to last measurement done at the oldest age of the follow up was found.

\section{Discussion}

Our results showed that all the valves are affected, but predominantly the mitral valve with a higher prevalence compared to the pediatric population $[2,3,5,12,13]$. The whole MV leaflets showed since the earlier stage, but more prominently in the latest stage, typical features such as stiffness and calcification involving the whole valvular apparatus, evolving in valve stenosis (Figure 2). In the latest stage, the most common valvular dysfunction was regurgitation in AV and TV. It was reported that in pediatric age the progression of the disease can show sometimes rapid progression $[2,3,5,12,13]$. Conversely, we found in our patients a slow progression, likely because they were under specific treatment. This result can also depend on the longer duration of the treatment and on a different rate of progression in pediatric age. Moreover, in the previous reports, it was not well specified whereas the patients were under treatment or not. 
Even though uncommon, echocardiography can help to avoid misdiagnose in adolescent and adult patients affected by attenuated form of MPS. Compared to other valvular diseases, such as rheumatic heart disease (RHD), the anterior mitral valve leaflet is fre- quently involved for the whole length since the earlier stage, while in the earlier stage of RHD only the marginal and mid parts of the leaflets are involved, with the classical doming and 'hockey stick' appearance and the calcifications are more extended. The pluri-

Table 1. General baseline characteristics.

\begin{tabular}{|c|c|c|c|c|c|c|c|}
\hline Pt number & Age & Gender & Type of MPS & Therapy & $\begin{array}{l}\text { Age start } \\
\text { therapy }\end{array}$ & $\begin{array}{l}\text { F/U yearsEcho studies } \\
\text { adolescent adult age }\end{array}$ & $\begin{array}{l}\text { Echo studies } \\
\text { adolescent adult }\end{array}$ \\
\hline 1 & 17 & M & VI & NO & l & 3 & 3 \\
\hline 2 & 45 & M & II & NO & I & 18 & 8 \\
\hline 3 & 16 & M & IV & ERT & 11 & 2 & 3 \\
\hline 4 & 15 & $\mathrm{~F}$ & VI & ERT & 6 & 1 & 2 \\
\hline 5 & 16 & $\mathrm{~F}$ & VI & BMT & 4 & 2 & 5 \\
\hline 6 & 19 & $\mathrm{~F}$ & IV & ERT & 11 & 5 & 5 \\
\hline 7 & 15 & $\mathrm{~F}$ & IV & ERT & 12 & 1 & 3 \\
\hline 8 & 17 & M & VI & BMT & 5 & 3 & 4 \\
\hline 9 & 20 & M & II & ERT & 17 & 4 & 2 \\
\hline 10 & 15 & $\mathrm{~F}$ & IV & ERT & 12 & +2 & 2 \\
\hline 11 & 19 & M & IV & ERT & 14 & 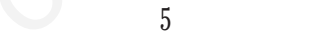 & 3 \\
\hline 12 & 48 & M & I & No & 1 & 18 & 1 \\
\hline 13 & 15 & $\mathrm{~F}$ & VI & ERT & 13 & 1 & 1 \\
\hline 14 & 15 & M & VI & ERT & 7 & 1 & 2 \\
\hline 15 & 32 & $\mathrm{~F}$ & VI & NO & / & 2 & 1 \\
\hline 16 & 16 & $\mathrm{~F}$ & VI & ERT & 5 & 2 & 1 \\
\hline 17 & 16 & M & VI & ERT & 5 & 2 & 2 \\
\hline
\end{tabular}

Table 2. Valve echocardiographic features adult adolescent age.

\begin{tabular}{lcccc}
\hline Valve & Thickening & Reduced motion & Diffuse calcification & Focal calcification \\
Anterior MV leaflet & $88 \%$ & $63 \%$ & $47 \%$ & $23 \%$ \\
Posterior MV leaflet & $86 \%$ & $70 \%$ & $41 \%$ & $35 \%$ \\
\hline Aortic valve & $76 \%$ & $23 \%$ & $23 \%$ & $59 \%$ \\
Tricuspid valve & $82 \%$ & $47 \%$ & $17 \%$ & $52 \%$ \\
\hline
\end{tabular}

Table 3. Valve dysfunction adult adolescent age.

\begin{tabular}{lccc} 
Valve & Stenosis & Regurgitation & Mixed stenosis regurgitation \\
Mitral & $29 \%$ & $23 \%$ & $23 \%$ \\
Aortic & $23.5 \%$ & $29 \%$ & $5 \%$ \\
\hline Tricuspid & $5 \%$ & $23 \%$ & $0 \%$ \\
\hline
\end{tabular}

Table 4. Valve thickness behavior.

\begin{tabular}{lccl}
\hline Valve thickness & Mean \pm STD baseline & Mean \pm STD last & p-value \\
AMVL (TIP) & $0.35 \pm 0.11$ & $0.39 \pm 0.09$ & $\mathrm{~ns}$ \\
AMVL (MID) & $0.31 \pm 0.057$ & $0.31 \pm 0.05$ & $\mathrm{~ns}$ \\
\hline PMVL & $0.29 \pm 0.166$ & $0.33 \pm 0.081$ & $\mathrm{~ns}$ \\
RCC & $0.21 \pm 0.06$ & $0.23 \pm 0.05$ & $\mathrm{~ns}$ \\
NCC & $0.185 \pm 0.05$ & $0.21 \pm 0.047$ & $\mathrm{~ns}$ \\
TV & $0.26 \pm 0.048$ & $0.27 \pm 0.05$ & $\mathrm{~ns}$ \\
\hline
\end{tabular}

Baseline, measurements at younger age in adolescence and adult age; last, measurements at the oldest age in adolescence and adult age. 


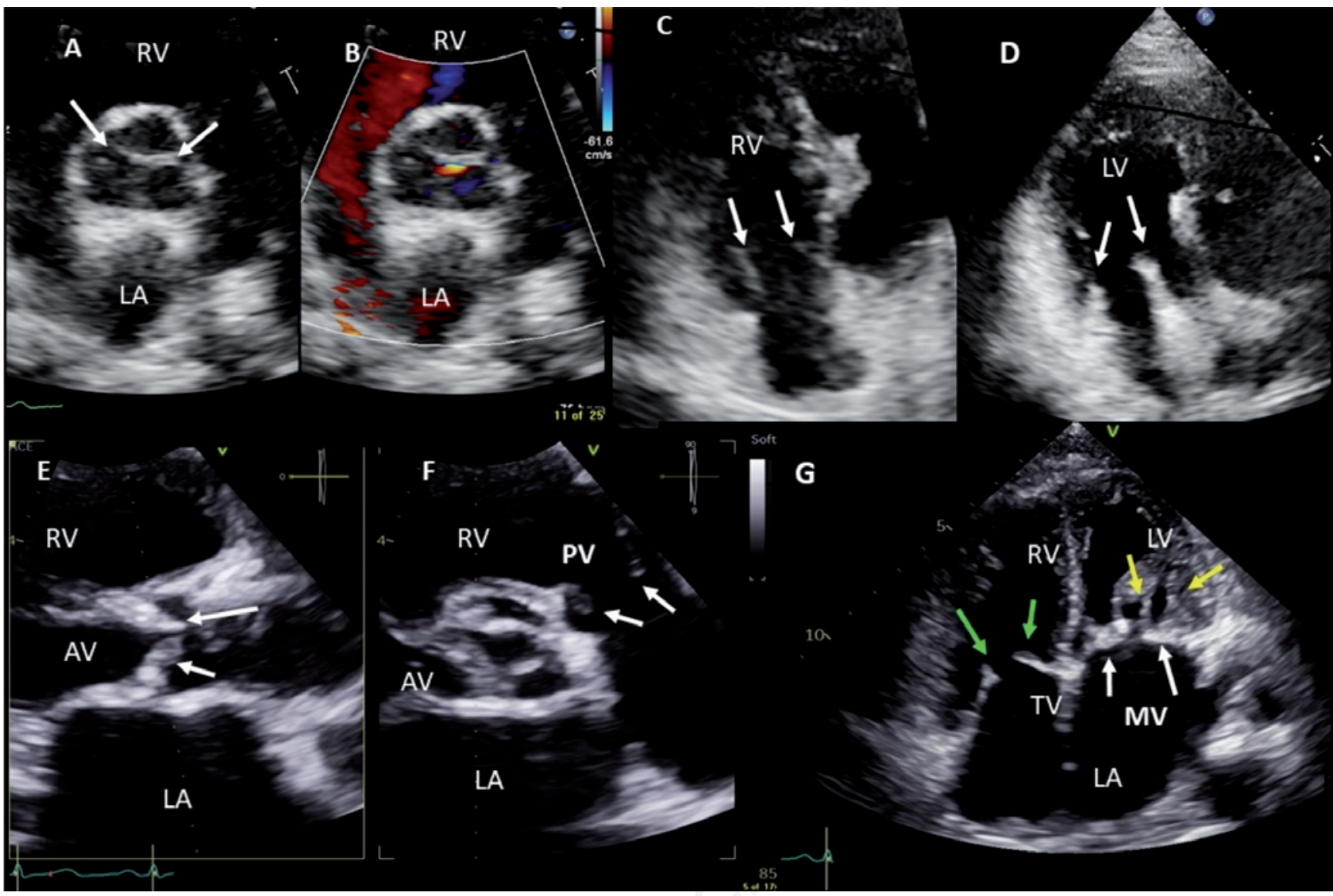

Figure 2. Transthoracic echocardiography. A-D) Mild valvular involvement involving the whole cusps and leaflets. A) Short axis at the level of aorta, mild thickening of the margins of the aortic cusps (white arrows). B) Short axis at the level of aorta: color doppler: mild central aortic regurgitation. C) Apical view for right ventricle mild thickening of tricuspid valve leaflets. D) Apical 3 chambers view: mild thickening of mitral valve leaflets. E-F) Significant involvement of four cardiac valves. E) Parasternal long axis, systole, stiffness, reduced motion, thickening and calcification of the aortic cusps. F) Short axis view at level of aorta and pulmonic valve, thickening of pulmonic valve cusps (white arrows). G) Apical 4 chambers view; important stiffness, reduced motion, thickening and calcification of mitral and tricuspid valve apparati (green arrows: tricuspid valve leaflets; white arrows: mitral valve leaflets; yellow arrows: mitral subvalvular apparatus showing severe fusion, matter and calcification). RV, right ventricle; LA, left atrium; LV, left ventricle; AV, aortic valve; $P V$, pulmonary valve; TV, tricuspid valve; MV, mitral valve.

valvular involvement as well as that of right sided valves is less frequent in RHD. The differential diagnosis with myxomatous valves is easier, being the prolapse never reported in MPS.

\section{Limitations}

Limitations of our study are the inherent limitations of any observational study, the small sample size and the length of follow up. However, being MPS a rare disease it is difficult to have a large number of patients with a longer follow up. We didn't report the data on pulmonic valve.

\section{Conclusions}

Our results showed that all the valves are affected, mainly the mitral valve with a higher prevalence compared to the pediatric age. The echocardiographic features of MPS differs from other valvular disease of adolescent and adult age, and knowing them can avoid misdiagnosis. Our observations also suggest that the progression of cardiac involvement slows after the initiation of the therapy in our group of age. Further studies on larger population are required to confirm our results.

\section{References}

1. Stapleton M, Arunkumar N, Kubaski F, et al. Clinical presentation and diagnosis of mucopolysaccharidoses. Mol Genet Metab 2018;125:4-17.

2. Parini R, Bertola F, Russo P. Molecular basis diagnosis and clinical management of mupolysaccharidoses. Cardiogenetics 2013;3:e2.

3. Boffi L, Russo P, Limongelli G. Early diagnosis and management of cardiac manifestations in mucopolysaccharidoses: a practical guide for paediatric and adult cardiologists. Ital $\mathbf{J}$ Pediatr 2018;44:122.

4. Russo P, Andria G, Baldinelli A, et al. [Il cardiologo e le 
mucopolisaccaridosi. Raccomandazioni del GICEM (Gruppo Italiano Cardiologi Esperti Malattie Metaboliche) su diagnosi, follow-up e management cardiologico].[Article in Italian]. G Ital Cardiol 2017;18:638-49.

5. Lin HY, Chuang CK, Huang YH, et al. Causes of death and clinical characteristics of 34 patients with Mucopolysaccharidosis II in Taiwan from 1995-2012. Orphanet J Rare Dis 2016;11:85.

6. Andrade MFA, Guimarães ICB, Acosta AX, et al. Left ventricular assessment in patients with mucopolysaccharidosis using conventional echocardiography and myocardial deformation by two-dimensional speckle-tracking method. J Pediatr (Rio J) 2019;95:475-81.

7. Baumgartner H, Falk V, Bax JJ, et al. 2017 ESC/EACTS Guidelines for the management of valvular heart disease. Eur Heart J 2017;38:2739-91.

8. Lang RM, Badano LP, Mor-Avi V, et al. Recommendations for cardiac chamber quantification by echocardiography in adults: an update from the American Society of Echocardiography and the European Association of Cardiovascular Imaging. J Am Soc Echocardiogr 2015;28:1-39.e14.
9. Sahasakul Y, Edwards WD, Naessens JM, Tajik AJ. Age-related changes in aortic and mitral valve thickness: implications for two-dimensional echocardiography based on an autopsy study of 200 normal human hearts. Am J Cardiol 1988;62:42430.

10. Webb RH, Culliford-Semmens N, Sidhu K, Wilson NJ. Normal echocardiographic mitral and aortic valve thickness in children. Heart Asia 2017;9:70-5.

11. Zoghbi WA, Adams D, Bonow RO, et al. Recommendations for noninvasive evaluation of native valvular regurgitation: A report from the American Society of Echocardiography developed in collaboration with the Society for Cardiovascular Magnetic Resonance. J Am Soc Echocardiogr 2017;30:303-71.

12. Çakar NE, Karaca M. Evaluation of echocardiographic findings of mucopolysaccharidosis cases. Eur Arch Med Res 2019;3:167-9.

13. Dangel JH. Cardiovascular changes in children with mucopolysaccharide storage diseases and related disorders-clinical and echocardiographic findings in 64 patients. Eur J Pediatr 1998;157:534-8. 